\title{
Arbitraje + Tecnología = Eficiencia, ¿dónde?
}

Andrea Espejo DonalRe

Abogada por la Universidad del Pacífico. Diplomado en Arbitraje Internacional y de las Inversiones en la Universidad del Pacífico.

SUMARIO:

I. Introducción.

II. Herramientas tecnológicas en el Arbitraje.

1. Audio y videoconferencia.

2. Recolección, revisión y producción de documentos.

3. Organización y transferencia de información.

4. Presentación de gráficos y evidencia, realidad virtual y realidad aumentada.

5. Herramientas de análisis y mapas conceptuales.

III. Seguridad de la Información.

IV. Conclusiones. 


\section{RESUMEN:}

En un mundo globalizado y constantemente evolucionando, el Arbitraje se encuentra en un punto esencial en su desarrollo, donde las tecnologías de la información están poco a poco introduciéndose y tomando un rol protagónico. La autora desarrolla cinco puntos clave donde la tecnología juega un papel esencial en el Arbitraje, no solo en el presente, sino también hacia donde se dirige en el futuro no tan lejano.

Palabras clave: arbitraje, tecnología de la información, inteligencia artificial, videoconferencias, IBA, Chartered Institute of Arbitrators, transferencia de información, discovery.

\section{ABSTRACT:}

In a globalized and ever-changing world, Arbitration is at a crossroads for its development, where the information technologies are slowly being introduced and taking a starring role. The author explores five key areas where technology plays an essential role in Arbitration, not only in the present, but also where it is headed in the near future.

Keywords: arbitration, information technology, artificial intelligence, videoconferences, IBA, Chartered Institute of Arbitrators, information transfers, discovery.
}

\section{INTRODUCCIÓN}

El atributo del Arbitraje que ha sido consistentemente más valorado $\mathrm{y}$, como contrapartida aquel que cada vez se echa más de menos, es la eficiencia a lo largo del proceso y la eficacia del resultado.' Eficiencia en términos de tiempo $y$ de costos; y la eficacia en cuanto a resultados concluyentes, o como otros los llamarían, resultados ejecutables.

En la búsqueda de una mayor eficiencia en los procesos arbitrales, se ha recurrido a múltiples mecanismos, como son: modernización de leyes de Arbitraje de los Estados, así como de reglamentos de múltiples instituciones arbitrales; nuevas reglas de soft law o guías sobre práctica de la prueba o conducta de las partes en el Arbitraje, como las que proporciona la International Bar Association - IBA- o la Cámara de Comercio Internacional $-\mathrm{CCl}-; \mathrm{y}$, por supuesto, la aparición de herramientas que se apoyan en la tecnología, las cuales son objeto de este artículo.
Así, considerando que una de las razones por las cuales las partes eligen el Arbitraje, en oposición a un proceso judicial, es la eficiencia, no sorprende que las partes que elijan el Arbitraje también sean propensas a favorecer un proceso más simplificado facilitado por herramientas tecnológicas. De esta manera, la flexibilidad es un elemento clave del Arbitraje que toma particular importancia al referirnos a la práctica de la prueba; y donde el empleo de la tecnología puede tomar un rol protagónico, como podremos ver más adelante.

Los soportes en tecnología, como softwares o programas online, están cada vez más presentes en la resolución de las disputas y, si son utilizados con regularidad, pueden convertirse en el principal aliado estratégico para lograr la tan reclamada eficiencia en los procesos arbitrales. ${ }^{2}$ De acuerdo con la encuesta publicada en el año 2018 por la Universidad Queen Mary y White and Case, la tecnología es generalmente utilizada en el Arbitraje Internacional y la mayoría de los encuestados prevé un mayor uso en el futuro en

1. "2018 International Arbitration Survey: The Evolution of International Arbitration", en Queen Mary University of London, acceso el 20 de marzo de 2021, http://www.arbitration.qmul.ac.uk/research/2018/.

2. Ibíd. 
lo que respecta a salas de audiencias digitales, almacenamiento de información en "la nube", videoconferencias e Inteligencia Artificial. ${ }^{3}$

Como es universalmente reconocido, la tecnología no es un nuevo socio de la industria legal en general ni del Arbitraje, pero está hoy más presente que nunca. ${ }^{4}$

El uso de la tecnología en la industria legal en general, y específicamente en el campo del Arbitraje, se puede dividir en dos grandes categorías.

Una primera categoría está referida a herramientas tecnológicas que no están involucradas en la decisión o predicción del resultado de un procedimiento legal y/o arbitral; sino que más bien se utilizan para aumentar la eficiencia del flujo de trabajo legal y/o arbitral. Los ejemplos en esta categoría incluyen firmas en línea, archivos electrónicos y videoconferencias.

La segunda categoría contiene herramientas para predecir, o incluso decidir el resultado de un procedimiento legal y/o arbitral. Los ejemplos en esta categoría incluyen Context by LexisNexis, una herramienta que se basa en el análisis de datos de decisiones anteriores respecto de una determinada materia, y a partir de dicha información proyecta posibles resultados con una probabilidad de acierto que puede superar el $70 \%$; otra herramienta diseñada para su uso en arbitrajes específicamente es Arbitrator Intelligence, que tiene como objetivo aumentar la transparencia, la rendición de cuentas y la diversidad en la selección de árbitros haciendo pública información clave sobre la toma de decisiones en el pasado por parte de árbitros. ${ }^{5}$
Lo que hay que anotar es que todas estas bondades han dejado de ser premonitorias, y se han convertido en realidades, de manera que al día de hoy permiten contar con procesos más eficientes o eficaces. Más eficientes en la medida que existen múltiples herramientas que reducen costos y tiempo en el proceso; y más eficaces, porque otras herramientas -o las mismaspermiten presentar un caso de una manera más directa y convincente a los árbitros.

Es por ello que este artículo tiene por objetivo centrarse en el empleo de la tecnología en el Arbitraje, como una herramienta actual y cercana, que promete otorgar múltiples ventajas a aquellos que practican el Arbitraje, y son usadas de manera más cotidiana y sencilla de lo que usualmente uno creería.

De esta manera, no se busca proporcionar una visión futurista de lo que podría llegar a lograrse con el empleo de la tecnología en el Arbitraje. Mucho se ha dicho, y seguramente se continuará diciendo, de cómo contribuirá en el futuro la tecnología, la neurociencia y la Inteligencia Artificial en el Arbitraje; pero poco se dice de como hoy las herramientas tecnológicas son una realidad, están a nuestro alcance y son capaces de impactar en la forma en la que se desarrollan los procesos arbitrales.

La mejor manera de aprovechar las herramientas que nos ofrece la tecnología es ponerlas en práctica, y para ello es indispensable conocer cuáles son aquellas que ya están a nuestra disposición. Dependerá de cada equipo adaptarse a estas novedades que nos proporciona la tecnología y plantear la estrategia que permita obtener el máximo provecho de estos instrumentos.

3. Ibíd.

4. Sophie Nappert citada por Liko Dokopoulou, "Arbitration X Technology: A Call For Awakening?", Kluwer Arbitration Blog, acceso el 20 de marzo de 2021, http://arbitrationblog.kluwerarbitration.com/2019/01/14/arbitration-x-technology-a-call-for-awakening/.

5. Catherine A, Rogers, "Arbitrator Intelligence: The Basics", Kluwer Arbitration Blog, acceso el 20 de marzo de 2021, http://arbitrationblog.kluwerarbitration.com/2018/02/27/ai-3/. 


\section{HERRAMIENTAS TECNOLÓGICAS EN EL ARBITRAJE}

A continuación, se presenta aquellas herramientas de tecnología actualmente disponibles en el mercado, muchas de ellas gratuitas, en función a las siguientes categorías: (i) Audio y videoconferencia; (ii) Recolección, revisión y producción de documentos; (iii) Organización y transferencia de información; (iv) Presentación de gráficos y evidencia, realidad virtual y realidad aumentada; (v) Herramientas de análisis y mapas concepciones.

Cabe destacar que el Subcomité IBA Arb40 ha elaborado una compilación de las ventajas tecnológicas que pueden ser usadas para asistir a aquellos que practican el Arbitraje Internacional, haciendo una división en ocho categorías, según lo siguiente:

\section{International Bar Association}

IBA Home About the IBA Committees and Divisions Outreach Membership Conferences and events IBA Digital Content

This committee's menu:

Select a category below for a summary of how the relevant technology might be used and several examples of vendors, software providers or companies that offer such technology.
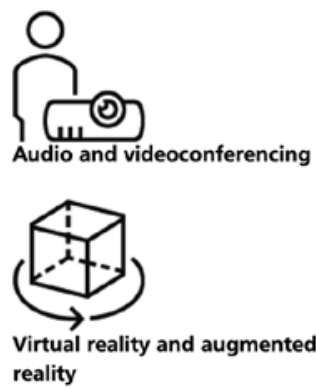

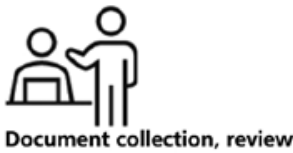
and production

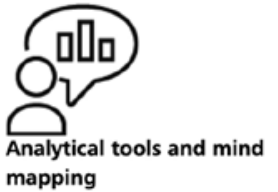

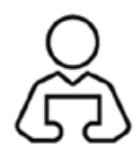

Management and transfer of arbitration data

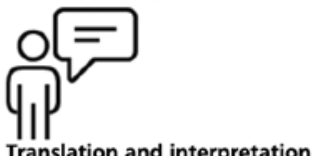

Translation and interpretation
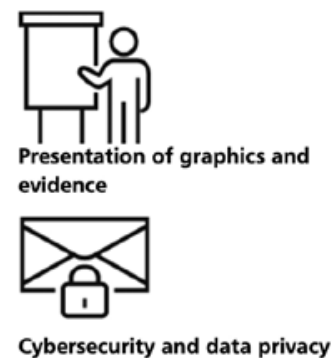

Lo que se presenta en las siguientes líneas toma parte de la información presentada por el Subcomité IBA, además de otras fuentes complementarias, comentarios y sugerencias para su empleo, según corresponda, en la búsqueda de proporcionar una visión práctica e incentivar el empleo de las mismas.

\section{Audio y videoconferencia.}

Esta es sin duda la primera categoría, en términos de antigüedad e importancia. El Arbitraje Internacional ha sido pionero en el uso de tecnología de audio e imágenes, tendencia que podemos ver con más frecuencia en los arbitrajes nacionales.

Gracias a esta tecnología, audiencias y otro tipo de coordinaciones entre partes y Tribunal, o entre árbitros, pueden desarrollarse de manera adecuada, pese a que los árbitros y/o las partes estén cada uno en lugares distintos. Esto permite ahorrar importantes costos para las partes y otorgarle gran celeridad al proceso. En esta materia tenemos herramientas de conference room como la plataforma de reuniones Bluejeans o la plataforma Opus 2 Magnum, que en su función básica es gratuita, siendo suficiente para conducir alguna audiencia vía conferencia telefónica o por videoconferencia, lo cual hace aún más real y cercana la comunicación.

Así, cada vez es más común que las audiencias se lleven a cabo por videoconferencia, considerando los altos costos que representa para las partes.

6. "Technology Resources for Arbitration Practitioners", en International Bar Association, acceso el 20 de marzo de 2021, https://www.ibanet.org/technology-resources-for-arbitration-practitioners.aspx. 
Sin embargo, incluso las videoconferencias continúan evolucionando, existiendo ya plataformas que permiten a las partes verse las unas a otras en 3D en tamaño real e incluso establecer contacto visual, este tipo de softwares viene siendo desarrollado por compañías como Cisco System.

\section{Recolección, revisión y producción de do- cumentos.}

En cuanto al manejo de información para efectos de un proceso de exhibición de documentos o discovery, en el marco de un arbitraje, cada vez es más frecuente la utilización de softwares que permiten efectuar una revisión general de gran volumen de información, pudiendo con ello identificar palabras o términos clave; separar documentos por categorías, en función a las necesidades y características de cada caso; y, transferir y almacenar gran cantidad de información.

Originalmente los procesos de discovery, propios del Common Law y adaptados en menor escala en el Arbitraje, han representado una gran cantidad de costos para las partes, no solo en costos de tiempo de abogados involucrados en la revisión de grandes volúmenes de información; sino también en relación a envío de la información y almacenamiento de la misma.

En la actualidad, se ha generado de esta manera lo que se denomina un "e-discovery", pues se intercambia la información solicitada por las partes a través de medios electrónicos. Cada vez más están disponibles soluciones tecnológicas que permiten que este proceso demande menor cantidad de tiempo y, en consecuencia, sea menos costoso, de manera tal que los costos relacionados a almacenar la información producida, así como el espacio físico para man- tenerlo, son ahora muy bajos, al punto de ser prácticamente irrelevantes financieramente.

Asimismo, se han desarrollado softwares que permiten la incorporación de la documentación y reducen el volumen de dicha data a través de herramientas que, por ejemplo, depuran aquellos que estén duplicados, pueden ser separados en categorías, a través del reconocimiento de caracteres, indexar documentos, identificación de palabras clave e identificación de documentos en otro idioma. Algunos ejemplos de estas plataformas son el software Relativity o Ringtail and Nuix.

Cabe destacar que en relación al e-discovery, instituciones arbitrales ya han emitido guías o protocolos para su aplicación, como son el Protocolo para "e-disclosure" en Arbitraje Internacional, elaborado por Chartered Institute of Arbitrators - ClArb de Londres ${ }^{7}$; o, el Reporte de la Comisión de la Cámara de Comercio Internacional de París "Managing E-Document Production".8 Por supuesto, estos documentos parten de las guías y directrices que ha sentado la IBA sobre práctica de la prueba; sin embargo, se enfocan de manera particular en la producción electrónica de documentos.

\section{Organización y transferencia de infor- mación.}

La primera herramienta por excelencia que podemos mencionar en esta categoría es el uso del correo electrónico. Es una realidad en el mercado arbitral peruano, el empleo del correo electrónico como un mecanismo ágil y poco costoso de comunicación y de compartir información. La utilización de esta herramienta con mayor naturalidad en el mercado peruano, como se hace desde hace muchos años en el Arbitraje Internacional, viene siendo propulsa-

7. "Protocol for E-Disclosure in International Arbitration", en Chartered Institute of Arbitrators, https://www.ciarb.org/media/1272/e-iscolusureinarbitration.pdf.

8. "ICC Commission Report - Managing E-Document Production", en International Chamber of Commerce, https://iccwbo.org/content/uploads/sites/3/2016/10/ICC-Arbitration-Commission-Report-on-Managing-E-Document-Production-2012.pdf. 
da por árbitros, instituciones arbitrales y partes sofisticadas.

El uso del correo electrónico no solo se limita a las coordinaciones y conversaciones habituales, sino que este se ha convertido en la vía de presentación de escritos o memoriales, o de presentación de posiciones respecto de alguna materia, que antes típicamente se habría hecho en una reunión o carta.

Si bien no nos estamos refiriendo a una plataforma compleja o no parece ser demasiado vanguardista, el impacto en términos de costo beneficio es impresionante. Correctamente utilizado reduce costos $y$, en especial, reduce una importante cantidad de tiempo, con la esperanza de que en el mercado local desaparezca la figura de "correr traslado", con todas las ineficiencias que ello genera, conduciéndonos más bien a una política de envíos y respuestas inmediatas, tanto entre las partes como por parte del Tribunal Arbitral.

Ahora bien, es cierto también que en muchos casos el uso del correo electrónico resulta insuficiente, pues se requiere realizar envío de una gran cantidad de información o se requiere organizar la data de determinada manera, para lo cual nuestros conocidos Outlook o Gmail resul$\tan$ insuficientes.

Bajo esos escenarios existen múltiples programas disponibles en el mercado que pueden funcionar como plataformas para almacenar, organizar y transferir una gran cantidad de información. Específicamente para el caso de arbitrajes internacionales, y cada vez más también para arbitrajes locales, sirven como una importante herramienta para la presentación y/o intercambio de memoriales entre las partes y con los árbitros; o con el propósito de que cada parte pueda contar con una base de datos lo suficientemente ágil y con la capacidad necesaria para albergar la gran cantidad de información que se suele producir en el marco de un proceso arbitral.

Las plataformas más básicas son sistemas basados en "la nube", servidores que sirven como repositorios de información, permitiendo a los usuarios compartir memoriales y pruebas con otros, a través de una única y centralizada locación a la que las partes y los miembros del Tribunal pueden acceder en cualquier momento. En este caso contamos como plataformas como BOX.COM, en la que se puede crear un espacio específicamente para un proceso, y dentro de la misma se puede reservar carpetas privadas para cada una de las partes y para el tribunal.

\section{Presentación de gráficos y evidencia, rea- lidad virtual y realidad aumentada.}

Sin duda el empleo de tecnología en la presentación de evidencia, tanto documental como respecto de testigos y expertos, es de los aspectos más interesantes y que debe buscar potencializarse más y emplearse con más frecuencia en los arbitrajes.

No podemos perder de vista que los árbitros son un panel al que se debe convencer, impactar y asegurar de que los argumentos queden claros y sean memorables. Por ello, la utilización de soporte tecnológico para lograr un informe oral efectivo o la actuación de una prueba, a través de ilustrar y resumir los argumentos, puede ser muy productivo.

La presentación de evidencia a través de herramientas de tecnología no solo brinda mayor realismo a la reconstrucción de los hechos en controversia, sino que incluso puede mejorar sustancialmente la dinámica de acreditar el caso a nivel técnico jurídico.

Sin duda, una presentación clara y contundente de los hechos materia de controversia es el elemento clave en la resolución exitosa de un arbitraje, y para ello se han desarrollado múltiples herramientas que buscan presentar de manera efectiva y visual la historia que las partes deben contar.

Por muchos años el empleo de Microsoft PowerPoint ha sido la principal herramienta utilizada en el Arbitraje para efectos del recuento de hechos y contar la historia que cada parte presenta frente a los árbitros, así como sus principales argumentos, de manera resumida y visual. Sin embargo, actualmente existen diversas con- 
sultoras que proveen asistencia con gráficos y presentaciones. Existen múltiples avances tecnológicos que listar, todos dirigidos a una experiencia visual/auditiva, que "dan vida" al caso en concreto, lo que se puede traducir en diseñar un caso sólido en argumentos, pero también sólido en persuasión.

Algunos ejemplos de herramientas que pueden emplearse para la realización de presentaciones que generan mayor impacto son: Impact trial, Prezi, RLM / TriaGraphix, SmartDraw, TrialPad - Lit Software, TrialTech, Z Axis. Muchas de estas plataformas son de acceso gratuito, al menos en una versión básica - pero que en muchas ocasiones resulta suficiente- o por un determinado tiempo.

También es posible encontrar herramientas para la presentación de documentos, por ejemplo durante el contra-interrogatorio de un experto o un testigo, como es el Trial director, por medio del cual se cuenta con funcionalidades para comparar documentos, resaltarlos, extraer alguna determinada sección, entre otros; que pueden ser particularmente útiles, dependiendo cómo se diseñe el contra-interrogatorio.

Asimismo, se han diseñado softwares para la presentación de testigos, por medio de los cuales se busca evitar que se emplee un número elevado de horas dedicadas a la preparación de un testigo, de manera previa a su participación en la audiencia en la que se actuará su declaración testimonial; sino que, a través de una serie de videos y brindando información, se logre sustituir la preparación cara a cara por parte de los abogados. Sobre esto contamos con la plataforma Perfect Witness, por ejemplo.

Por supuesto, no consideramos que estas plataformas estén diseñadas para sustituir al 100\% la labor de preparación que hacen los abogados a través de simulaciones, pero sí pueden ser muy útiles para ahorrar un número importante de horas de abogados y procurar que el testigo se sienta más preparado y familiarizado con lo que ocurrirá en la audiencia.

Ahora, en los últimos años, además de los pro- gramas antes mencionados que han sido diseñados para crear presentaciones más persuasivas, se han desarrollados altas tecnologías, como las siguientes: (i) simulaciones tridimensionales 3D; (II) realidad aumentada, que emplea elementos del mundo real con facilidades tecnológicas animadas; (iii) realidad virtual, que replica artificialmente un determinado ambiente.

En cualquiera de estos tres casos ya nos enfrentamos a herramientas mucho más sofisticadas, costosas y que requieren de especialización. Así, existen consultoras especializadas en la creación de simulaciones, que son utilizados en juicios o arbitrajes para representar como aconteció -o debió haber acontecido- determinado hecho, siendo poco probable que una parte -o sus abogados - se desenvuelvan con naturalidad en este tipo de programas. Al respecto, algunos proveedores o programas que pueden emplearse son los siguientes: A2L Consulting, Cogent Legal, Courtroom Visuals, DAQRI Worksense Model.

En cuanto a realidad virtual, cabe destacar que este es un mercado relativamente nuevo y no hay aún una práctica significativa en materia de Arbitraje. Esta tecnología permite la creación de ambientes que son artificiales, interactivos, creados por computadora y con gráficos en 3D, lo cual da al usuario la experiencia de estar presente en un ambiente virtual. Por ejemplo, reconstruir un accidente en el marco de un contrato de construcción o la explicación de efectos en la contaminación ambiental.

Como se señaló, esto parece particularmente útil en la actuación de pruebas en controversias cuyos hechos son un componente importante para resolver la disputa. Es una realidad que los arbitrajes los ganan aquellos que acreditaron que los hechos están a su favor. Emplear herramientas como la realidad virtual puede devenir en convencer al Tribunal de que los hechos se desarrollaron de determinada manera y eso deriva en qué parte tiene la razón.

\section{Herramientas de análisis y mapas con- ceptuales.}

En los procesos arbitrales, en general, y particu-

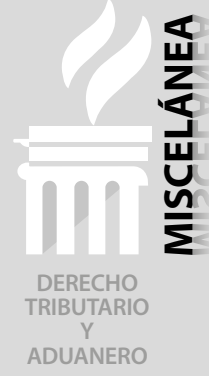

. 
lamente en algunos de ellos, la cantidad de información y argumentos que se debe manejar es muy amplia. En muchos casos, el volumen de información puede volverse difícil de manejar, de procesar y aún más de explicar, trasladar y convencer a los árbitros.

En ese sentido, se han desarrollado herramientas de análisis, las mismas que consisten en nuevas aplicaciones que pueden ayudar a los abgados de las partes, e incluso a los árbitros, a desarrollar y explorar hechos y aspectos legales, así como organizarlos de manera lógica, usualemente visual.

Estas herramientas analíticas pueden usarse como mapas de estrategias, patrones, relaciones, prioridades, tendencias y tiempo a ser empleado para resolver una determinada disputa. A partir de ello, se logra dirigir la atención del público al que está dirigido a los aspectos más importantes del caso, y presentarlos de manera lógica, ordenada y visual, de manera que se logre el objetivo último que es convencer respecto de determinada posición.

En línea con ello, se han podido generar otros avances, como el denominado mind-mapping, lo cual consiste en que es posible efectuar predicciones acertadas en cuanto al resultado de un caso particular a partir del análisis de información pasada. Por ejemplo, un programa que era alimentado por una serie de decisiones adoptadas por la Corte Europea de Derechos Humanos fue capaz de predecir el resultado de otros casos con el $79 \%$ de certeza, en función de buscar relación entre palabras, secuencia de palabras y palabras clave que derivan en un resultado o en otro. ${ }^{9}$

Por tanto, las herramientas como las antes señaladas son útiles para el global entendimiento de una controversia, para la generación de una estrategia adecuada y para determinar la forma más asertiva de canalizarlo; así como para predecir con moderada certeza posibles resultados. Sobre esto punto, tenemos plataformas como Brainspace, LexMachina, Courtroom Visuals, Ross Intelligence o TreeAge Pro.

\section{SEGURIDAD DE LA INFORMACIÓN}

Un comentario final, transversal a todas las herramientas presentadas, es lo referido a la seguridad de la información. Sin lugar a dudas lo relacionado a data privacy y cyberseguridad ha dado espacio a amplias discusiones entre los practicantes de la industria, que escapan del objeto de lo que aquí se trata; sin embargo, al emplear las herramientas aquí presentadas es importante tener en mente que la seguridad de la información intercambiada en el marco de un arbitraje es un tema mayor.

Fundamentalmente, la discusión se ha centrado en proteger la información que es intercambiada entre las partes y los árbitros, y garantizar que las plataformas que para ello se empleen brinden la seguridad suficiente respecto de la documentación generada.

Actualmente, no existe ningún estándar perfecto de cybersegridad en general; no solo en materia legal o en relación al Arbitraje. En reemplazo de ello, varias instituciones arbitrales, organizaciones y practicantes del Arbitraje, incluyendo la IBA, han desarrollo protocolos o directrices con el objetivo de prevenir que los practicantes del Arbitraje sean víctimas de las debilidades típicas y busquen emplear de forma cotidiana aquellas medidas que pueden protegerlos en contra de estas debilidades.

El nivel apropiado de protección de la privacidad y seguridad de la información depende la naturaleza del arbitraje, la sensibilidad de la información intercambiada y la norma aplicable; y puede derivar en que se añadan determinados costos al proceso, en búsqueda de mayores candados de seguridad.

\section{CONCLUSIONES}

En resumen, hemos visto hasta este punto los 
distintos aspectos prácticos en los que contribuye la tecnología para llevar adelante un proceso arbitral más eficiente, ya sea porque representa menores costos, menor inversión de tiempo, mayor efectividad, o más de uno de los anteriores beneficios.

El uso de las herramientas de tecnología en el contexto del Arbitraje, en especial en el Arbitraje Internacional, es no solo cada vez más frecuente, sino que se ha convertido en un aliado indispensable para llevar adelante procesos arbitrales que, en algunos casos, por su vocación auténticamente internacional, requieren de instrumentos que faciliten el desarrollo eficiente del proceso; o, simplemente porque requerimos cada vez de más y mejores herramientas que permitan llevar un caso de manera más eficiente o presentarlo de forma más eficaz.

Siendo que una de las principales razones por las que las partes optan por el Arbitraje, en reemplazo de un proceso judicial es la eficiencia, no resulta sorprendente que esas mismas partes sean más proclives para emplear herra- mientas o facilidades tecnológicas a lo largo del proceso. Sin embargo, se puede observar en el mercado actual que tanto las partes como sus abogados tienden a ser altamente adversos al riesgo y ello se extiende a ser menos propensos a experimentar con el uso de nuevas tecnologías.

Muchas son las herramientas de tecnología que se pueden emplear para presentar determinadas pruebas, o para plantear argumentos, o presentar el caso en general; sin embargo, los árbitros no entenderán el caso que tienen frente a ellos si no se les provee con los instrumentos adecuados, en términos de presentación y de argumentación. Así, las herramientas de tecnología que se empleen serán tan confiables como la data que está detrás de ellas y las soportan.

Todas estas herramientas, por más interesantes y persuasivas que hayan sido diseñadas, pierden propósito sin una estrategia clara detrás, la cual hasta la fecha no puede ser desarrollada por una inteligencia distinta a la humana. 